\title{
Magneto-Inertial Fusion
}

\author{
G. A. Wurden ${ }^{1}$ - S. C. Hsu ${ }^{1}$ - T. P. Intrator ${ }^{1}$ T. C. Grabowski ${ }^{2}$ J. H. Degnan ${ }^{2}$ ' \\ M. Domonkos ${ }^{2}$ - P. J. Turchi ${ }^{3}$ E. M. Campbell ${ }^{4}$ - D. B. Sinars ${ }^{4}$ - M. C. Herrmann ${ }^{5}$. \\ R. Betti ${ }^{6}$ B. S. Bauer ${ }^{7}$ I. R. Lindemuth ${ }^{7}$ - R. E. Siemon ${ }^{7}$ - R. L. Miller ${ }^{8}$. \\ M. Laberge ${ }^{9} \cdot$ M. Delage ${ }^{9}$
}

Published online: 17 November 2015

(C) The Author(s) 2015. This article is published with open access at Springerlink.com

\begin{abstract}
In this community white paper, we describe an approach to achieving fusion which employs a hybrid of elements from the traditional magnetic and inertial fusion concepts, called magneto-inertial fusion (MIF). The status of MIF research in North America at multiple institutions is summarized including recent progress, research opportunities, and future plans.
\end{abstract}

Keywords Magneto-inertial fusion $\cdot$ Magnetized target fusion · Liner · Plasma jets · Fusion energy · MagLIF

\section{Description}

Magneto-inertial fusion (MIF) (aka magnetized target fusion) [1-3] is an approach to fusion that combines the compressional heating of inertial confinement fusion (ICF) with the magnetically reduced thermal transport and magnetically enhanced alpha heating of magnetic confinement

G. A. Wurden

wurden@lanl.gov

Los Alamos National Laboratory, Los Alamos, NM, USA

2 Air Force Research Laboratory, Albuquerque, NM, USA

3 Santa Fe, NM, USA

4 Sandia National Laboratory, Albuquerque, NM, USA

5 Lawrence Livermore National Laboratory, Livermore, CA, USA

6 University of Rochester, Rochester, NY, USA

7 University of Nevada Reno, Reno, NV, USA

8 Decysive Systems, Santa Fe, NM, USA

9 General Fusion, Vancouver, Canada fusion (MCF). From an MCF perspective, the higher density, shorter confinement times, and compressional heating as the dominant heating mechanism reduce the impact of instabilities. From an ICF perspective, the primary benefits are potentially orders of magnitude reduction in the difficult to achieve $\rho r$ parameter (areal density), and potentially significant reduction in velocity requirements and hydrodynamic instabilities for compression drivers. In fact, ignition becomes theoretically possible from $\rho r \leq 0.01 \mathrm{~g} / \mathrm{cm}^{2}$ up to conventional ICF values of $\rho r \sim 1.0 \mathrm{~g} / \mathrm{cm}^{2}$, and as in MCF, $\mathrm{Br}$ rather than $\rho r$ becomes the key figure-of-merit for ignition because of the enhanced alpha deposition [4]. Within the lower- $\rho r$ parameter space, MIF exploits lower required implosion velocities $(2-100 \mathrm{~km} / \mathrm{s}$, compared to the ICF minimum of $350-400 \mathrm{~km} / \mathrm{s}$ ) allowing the use of much more efficient $(\eta \geq 0.3)$ pulsed power drivers, while at the highest (i.e., ICF) end of the $\rho r$ range, both higher gain $G$ at a given implosion velocity as well as lower implosion velocity and reduced hydrodynamic instabilities are theoretically possible. To avoid confusion, it must be emphasized that the wellknown conventional ICF burn fraction formula does not apply for the lower- $\rho r$ "liner-driven" MIF schemes, since it is the much larger mass and $\rho r$ of the liner (and not that of the burning fuel) that determines the "dwell time" and fuel burnup fraction. In all cases, MIF approaches seek to satisfy/ exceed the inertial fusion energy (IFE) figure-of-merit $\eta G \sim 7-10$ required in an economical plant with reasonable recirculating power fraction. A great advantage of MIF is indeed its extremely wide parameter space which allows it greater versatility in overcoming difficulties in implementation or technology, as evidenced by the four diverse approaches and associated implosion velocities shown in Fig. 1.

MIF approaches occupy an attractive region in thermonuclear $\rho-T$ parameter space, as shown in a paper by 


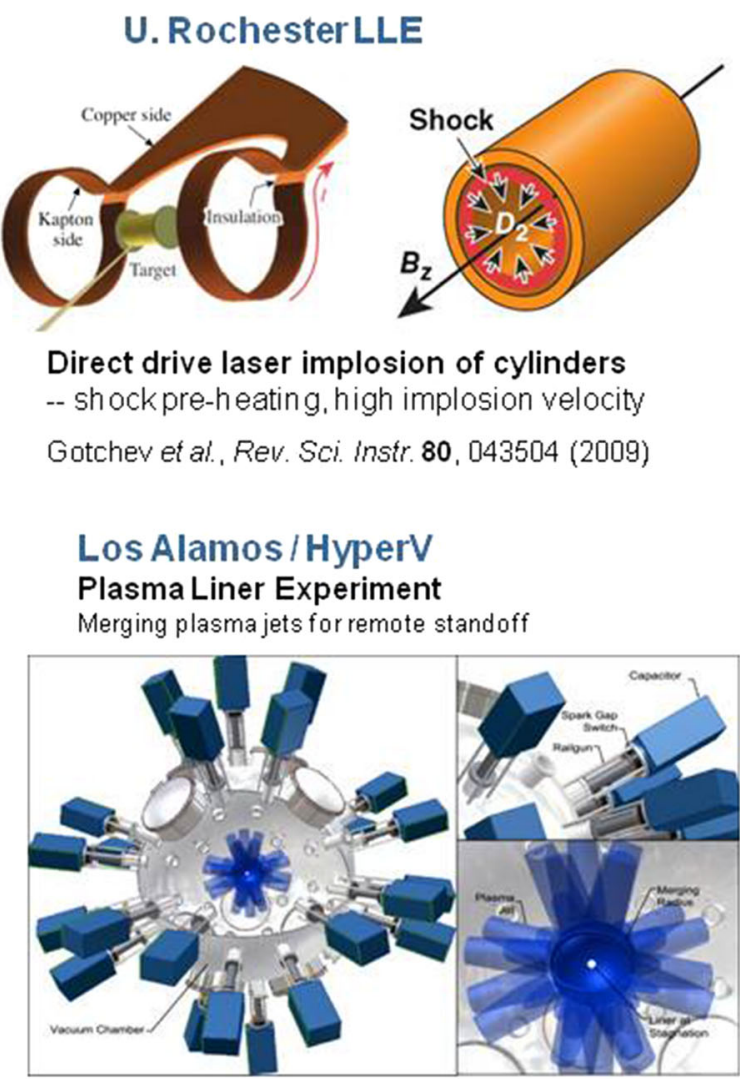

S. C. Hsu et al., IEEE Trans. Plasma Sci. 40, 1287 (2012)

\section{Los Alamos / AFRL \\ Field Reversed \\ Configuration \\ Shiva Star FRCHX \\ $\sim 20 \mu \mathrm{s}, 0.5 \mathrm{~cm} / \mu \mathrm{s}$ liner implosion}

Taccetti, Intrator, Wurden et a Rev. Sci, instr. 74, 4314 (2003) Degnan et al., IEEE Trans. Plas. Sci 36, 80 (2008)

\section{Sandia National Laboratories}

Magnetized Liner Inertial Fusion

Laser preheated magnetized fuel

LASNEX simulations in dicate interesting yields
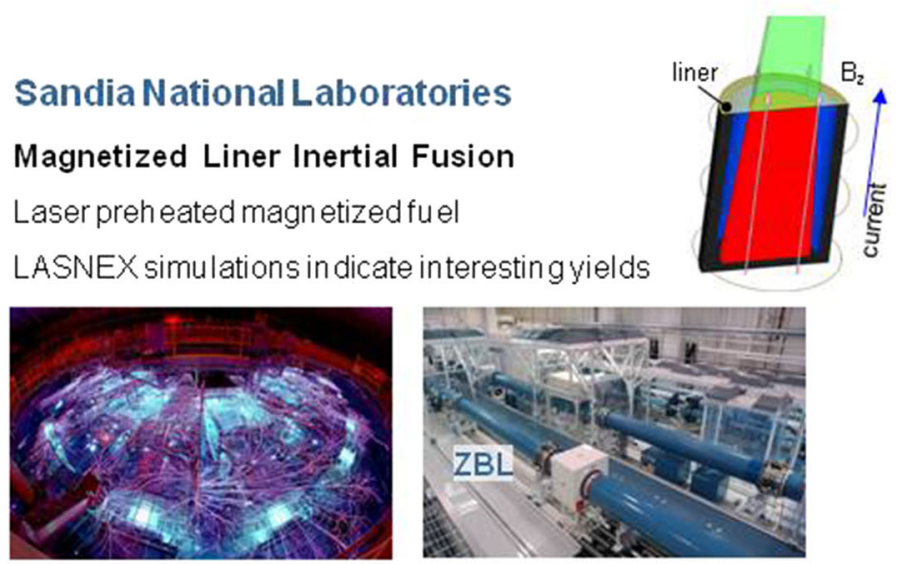

S. A. Slutz, et al., Phys. Plasmas 17, 056303 (2010)

Fig. 1 Many of the MIF concepts presently being explored in the USA

Lindemuth and Siemon [3] from physics first principles. The center of the attractive region is at a density value that is approximately the geometric mean of ICF and MCF. A key point here is that burning plasma class MIF driver facilities, which already exist (e.g., Z/Z-Beamlet, or perhaps ATLAS), cost $\leq \$ 200 \mathrm{M}$ compared to the multi- $\$ \mathrm{~B}$ ITER and NIF. These existing facilities can address much of the physics critical to MIF concepts that are required for large fusion yields and system gain. For this reason alone, MIF warrants serious attention. Furthermore, the density regime of MIF is in a relatively unexplored area of magnetized plasma physics and plasma/material interactions, thereby allowing a multitude of opportunities in plasma science frontiers.

\section{Status}

The USA is a world leader in MIF research. In the last 10 years, there have been substantial advances and growing interest in MIF research and concepts. A team led by Los Alamos National Laboratory (LANL) and the Air Force Research Laboratory (AFRL) has been investigating solid liner compression of magnetically confined field-reversed configuration (FRC) plasmas to achieve kilovolt temperatures [5-7]. The University of Rochester has introduced seed magnetic fields into the center of targets at the OMEGA laser facility, and compressed those fields by imploding a liner with the OMEGA laser. They have obtained record values of magnetic field and demonstrated increases in neutron yields [8-10]. Sandia is developing magnetized liner inertial fusion (MagLIF), in which a magnetically driven beryllium liner, imploded by the Z-machine, adiabatically compresses a laser-preheated magnetized DT target plasma [11-14]. In the very first series of integrated MagLIF shots last year, $>10^{11}-10^{12}$ DD neutrons were observed, indicating significant improvement in target performance due to the presence of preheated and magnetized fuel in the target [15]. The experiments also showed a significant DT/DD ratio $\left(\sim 10^{-2}\right)$ from a pure $D_{2}$ fuel indicating magnetization of the DD fusion produced tritons [16] with an estimated $\mathrm{B} * \mathrm{R}$ product of $0.4 \mathrm{MG}-\mathrm{cm}$ (which was also separately inferred from DT neutron time of flight measurements). LANL also leads a team that is exploring a standoff concept of using a spherically convergent array of gun-driven plasma jets to 
achieve assembly and implosion of a plasma liner (PLX) without the need to destroy material liners or transmission lines on each shot [17-20]. A private company, general fusion (GF) in Canada, with many Americans working for it, is developing a merging compact toroid plasma source and envisions repetitively fired acoustic drivers that would drive a liquid liner compression of a magnetized target [21-23].

Much of the current MIF work can be traced back, at least in part, to work on imploding liners for controlled fusion at the Kurchatov Institute of Atomic Energy, under E. P. Velikhov, circa 1970 [24]. This inspired the Linus project at the Naval Research Laboratory [25], and later the fast-liner project at Los Alamos [26]. In Russia, MIF took a form called magnitnoye obzhatiye, or magnetic compression (MAGO), first revealed by Russian scientists when the Cold War ended [27-29], and worked on collaboratively with experiments at LANL [30]. Presently the USA clearly holds world leadership in MIF research, but fledgling MIF efforts are also underway in China and France. Russia has also stated that it is constructing a pulsed power facility at twice the current ( $\sim 50 \mathrm{MA})$ and four times the delivered energy to the load compared to $\mathrm{Z}$ to explore MIF concepts. These approaches span implosion time scales ranging from ns to hundreds of $\mu$ s and all have substantially different "target physics" issues.

\section{Current Research and Development (R\&D)}

\section{R\&D Goals and Challenges}

An MIF grand challenge is to determine and quantitatively understand how driven or self-generated magnetic fields can facilitate ignition or increase yield for a variety of inertial fusion schemes. For the wide range of plasma compression strategies there are several overarching physics goals that must be addressed. These include (1) whether suitable target plasmas can be formed and subsequently compressed and heated to thermonuclear temperatures while avoiding high $\mathrm{Z}$ contaminants; (2) what are the transport mechanisms for particle, energy, and magnetic flux losses; and (3) characterization of the plasma boundary interface and the robustness and stability of initial target configurations. Each of these broad topics involves engineering and basic science components that overlap conventional MFE and IFE concerns. Since one major justification for pursuing MIF invokes simpler and less expensive implementations compared with conventional fusion approaches, practical cost considerations should be not be overlooked. As with ICF schemes, the cost of material that must be recycled versus consumed for each pulse (the "kopeck" problem) is an important issue.

\section{Related R\&D Activities}

MIF reactor systems tend toward larger yields and lower repetition rates than conventional unmagnetized ICF, and most likely as a result will need to (and are able to) use liquid-walled chamber systems, which are also relevant for other ICF targets and drivers especially heavy-ion beam driven fusion. Liquid "fusion facing" walls have the potential to significantly reduce the "first wall" material challenges common for most mainline approaches to fusion energy. Present MIF work falls under the category of Magnetized High Energy Density Laboratory Plasmas, and its science is well documented in the recent FESAC HEDLP Basic Research Needs Report (2010) and the National Academy of Sciences Inertial Confinement Fusion report (2013).

\section{Recent Progress}

At Rochester LLE, a fusion yield enhancement due to a compressed magnetic field that was externally introduced into the fusion fuel prior to laser-driven implosion has been unequivocally demonstrated experimentally using the OMEGA laser. The results are consistent with 1-D modeling estimates. In spherical implosions of solenoidal (axial) magnetic field with open field lines, a statistically significant neutron yield increase of $30 \%$ was obtained, and proton deflectometry measured a compressed magnetic field of 23 Megagauss in similar spherical implosions. If magnetic field with closed field lines could be introduced in the same target plasma, a factor of 2 to 4 increase in neutron yields is expected. In previous cylindrical implosions, magnetic field in excess of 70 Megagauss was detected. In all of these experiments the initial applied axial magnetic field is $\sim 10 \mathrm{~T}(0.1 \mathrm{MG})$. The density in these experiments is not optimum but serves as an example of the wide range of densities over which MIF might operate. This early success has motivated research to explore the impact of magnetic fields on a range of targets at the National Ignition Facility at Livermore [31].

A deformable liner system has been developed and tested at the Air Force Research Laboratory (AFRL) on Shiva Star, and a field-reversed configuration (FRC) plasma target has been developed at Los Alamos and ported to AFRL. The experiments are based on early work on compression of an FRC by an explosively driven liner [32], but to avoid shocks and have a continuously increasing liner velocity during the implosion, an electromagnetically driven liner is used instead. The AFRL/ LANL experiments were guided with extensive modeling, from plasma formation through liner compression, by NumerEx, LLC using MACH2. The first integrated plasma/liner engineering test of the Field-Reversed 
Configuration Heating Experiment, or FRCHX, on Shiva Star was performed in April 2010, but for this test the plasma lifetime was too short compared to the compression time $(23 \mu \mathrm{s})$. After extensive diagnostic studies and a series of improvements were implemented, most notably the inclusion of a longer capture region, the lifetime of trapped flux within the FRC was improved such that it was now comparable to the implosion time [33], and an integrated compression test was conducted in Oct. 2013. The FRC was compressed cylindrically by more than a factor of ten, with density increasing more than 100 -fold, to $>10^{18} \mathrm{~cm}^{-3}$ (a world FRC record), but temperatures were only in the range of $300-400 \mathrm{eV}$, compared to the expected several $\mathrm{keV}$. Although compression to Megabar pressures was inferred by the observed time and rate of liner rebound, we learned that the heating rate during the first half of the compression was not high enough compared to the normal FRC decay rate. Principal diagnostics for this experiment were soft X-ray imaging, soft X-ray diodes, and neutron measurements. LANL/AFRL has developed a new proposal, not yet funded, to use double-sided FRC injection and trapping, with $5 \mathrm{~T}$ initial fields, to address these issues.

The 80-terawatt $\mathrm{Z}$ facility at Sandia National Laboratories is the world's largest stationary pulsed power facility, capable of generating up to 26 million Amperes of current in a $\sim 100 \mathrm{~ns}$ pulse. These large currents can be used to create large magnetic fields $(\sim 5000 \mathrm{~T})$ and pressures $(\sim 100 \mathrm{Mbar})$ in $\mathrm{mm}$-scale targets. The $\mathrm{Z}$ facility supports a wide variety of stockpile stewardship and basic high energy-density experiments, including measuring the equation of state of materials under extreme conditions, developing intense radiation sources, and inertial confinement fusion research. The particular form of magneto-inertial fusion being tested at the $\mathrm{Z}$ facility is a relatively new concept known as magnetized liner inertial fusion (MagLIF). Sandia $Z$ experiments and 2D and 3D modeling have begun, with NNSA support. MagLIF uses a small, low aspect ratio liner (outer radius/liner thickness is $\sim 6$ ) beryllium liner to compress a laser-initiated axial plasma embedded in an axial magnetic field. In the MagLIF concept, a magnetically imploded, cylindrical metal liner is used to compress fusion fuel that has been magnetized by an externally applied axial field (10-30 T) and preheated to $\sim 100-300 \mathrm{eV}$ using a laser (other preheating concepts are also being explored). Simulations indicate it is possible to achieve $100 \mathrm{~kJ}$ DT fusion yields on the $\mathrm{Z}$ facility, a yield comparable to the energy coupled to the fusion fuel, at final fuel pressures of about 5 Gbar. To do this will require a 26-MA drive current, about $6-10 \mathrm{~kJ}$ of $0.532 \mu \mathrm{m}$ laser energy delivered over 8-10 ns, an applied magnetic field of $30 \mathrm{~T}$, and DT fuel. Scaling studies suggest that high-yield ( $\sim 1$ GJ), high-gain ( $>100)$ targets may be possible on a future $>61$ MA pulsed power facility using similar preheat and magnetic field parameters. A smaller facility ( 47 MA) could produce fusion yields from volume burning DT in the 5-10 MJ range. Further research progress with $\mathrm{Z}$ experiments is essential for moving forward.

Over the past year, the first fully integrated MagLIF experiments were conducted using deuterium fuel. The drive current was 18-20 MA and external field coils delivered up to $10 \mathrm{~T}$ magnetic fields over a several $\mathrm{cm}^{3}$ volume. Meanwhile, the Z-Beamlet laser irradiated a $\sim 3 \mu \mathrm{m}$ thick foil covering the laser entrance hole in the liner, delivering $2-2.5 \mathrm{~kJ}$ of laser energy in about $2 \mathrm{~ns}$ to ionize the gas fill. The foil is necessary to keep the $0.8 \mathrm{mg} / \mathrm{cc} \mathrm{D}_{2}$ gas in the Be liner. Off line experiments showed that only 100-300 J of laser energy was transmitted through the foil to preheat the fuel. These experiments produced significant DD fusion yield $\left(\sim 5 \times 10^{11}-2 \times 10^{12}\right.$ neutrons), high ion temperatures $(>2-2.5 \mathrm{keV})$, high electron temperatures $(\sim 3.5$ $\mathrm{keV}$ ), and significant secondary $14.1 \mathrm{MeV}$ neutrons arising from triton burn-up [16]. Additional imaging and time resolved x-ray measurements show strong stagnation of the fusion fuel-all occurring with implosion velocities of $\sim 70 \mathrm{~km} / \mathrm{sec}$. The data is consistent with significant flux compression and magnetized electrons and tritons. MagLIF in its current configuration with external field coils for magnetization and a large laser for fuel heating may not be immediately obvious as an energy platform, but it may be well suited to quickly determining the promise of magnetoinertial fusion. Indeed, the initial results are promising in that these targets do not work by traditional ICF metrics that require high velocities and high $\rho$-R.

To test the possibility of a standoff driver [34, 35] (one without physical leads to the liner thus avoiding repetitive hardware destruction), a plasma liner formed from multiple plasma jets $[17,18]$ is being pursued again at LANL, i.e., plasma-jet-driven MIF or PJMIF, funded by an ARPA-E for the next 3 years under its accelrating low-cost plasma heating and assembly (ALPHA) program. A 2.7-meter diameter spherical vacuum chamber is the centerpiece of the plasma liner experiment (PLX) at LANL, which has also conducted basic plasma shock experiments $[19,20,36,37]$ using two plasma railguns that were developed by HyperV Technologies Corporation. The PLX team will conduct 36-60 coaxial-gun experiments that aim to address the key MIF-relevant scientific issues of spherically imploding plasma liners as a standoff driver. The near-term objectives of plasma liner experiments under ARPA-E sponsorship will be to (1) demonstrate for the first time the formation of a spherically imploding plasma liner via an array of merging plasma jets, (2) obtain experimental data on the scaling of peak liner ram pressure with initial plasma jet parameters, and (3) characterize liner uniformity and explore methods to control uniformity.

The Canadian private company General Fusion has been exploring the compression of spheromak plasmas via 
sonically driven shock waves into a fluid lead-lithium liner. The company has constructed and tested elements of their acoustic system, achieving milestones for the energy input (125 kJ/piston) and timing control required on their driver $( \pm 5 \mu \mathrm{s})$. General Fusion has a test stand with 14 pistons arranged around a spherical chamber. This is insufficient to achieve symmetric implosion. It was built to gain practical experience with pumping liquid lead, forming a vortex, firing many servo controlled pistons, etc. Depending on the confinement achieved during compression and the size of the pistons, the final system will require 200-400 pistons. General Fusion is also operating a relatively large $(100 \mathrm{~kg} / \mathrm{s})$ molten lead loop for liner formation. They have successfully injected $200-300 \mathrm{eV}$ magnetized spheromak plasmas into their capture region, and kept these plasmas confined there for over $500 \mu \mathrm{s}$, more than $3 \times$ the implosion timescale. Most recently, they have begun high-explosive driven liner tests at a contractor facility. The high explosive driven liners used solid aluminum liners initially coated using titanium gettering to reduce impurity influx. These ongoing tests are clearly different than the liquid lead-lithium planned for repeatable liquid compression, but allow an early single-shot approach to high energy compression testing. During compression, only a $3 \times$ increase of the initial magnetic field was observed. Analysis indicates this disappointing result was most likely due to plasma impurity problems. These impurity problems (due to delamination of titanium coatings on the inside surface of the liner) are being mitigated with lithium coatings. While no measurable neutron yields have been achieved to date, work is continuing, and the next round of venture capital funding has been secured.

\section{Budget}

Historically, MIF budgets under DOE fusion energy sciences (FES) auspices were recently as large as $\$ 7 \mathrm{M}$ per year nationally, out of a $\$ 25 \mathrm{M} /$ year HEDLP effort. Due to recent FES reprioritization towards ITER and tokamaks in FY14, this funding level has been zeroed. Given the potential of MIF, many scientists and engineers would like to see this decision reversed, so that FES continues to steward MIF research, even at Discovery Science levels. Recently (2014), DOE's ARPA-E office announced a \$30 M solicitation entitled "accelerating low-cost plasma assembly and heating (ALPHA)" to focus on developing low-cost tools to enable rapid learning and higher shot rate toward faster fusion energy development. Announcements of 9 awards occurred on May 14, 2015 [38].

\section{Anticipated Contributions}

- Energy concepts-Given the limited funding, the longterm application of MIF to energy production has not been examined at a systems level as extensively as conventional magnetic or inertial fusion, and the metrics are less well defined. At a high level, with MIF, yields in the gigajoule range would allow operation at a lower repetition rate than conventional ICF, though the PJMIF concept is somewhat intermediate and aims for yields well below $1 \mathrm{GJ}$ but with a $\sim 1 \mathrm{~Hz}$ repetition rate. Physics challenges in designing and testing target concepts that can achieve these fusion yields and gain have been identified. Much of the work on recyclable transmission lines contained in the Z-IFE 4 year reactor design effort, led by Sandia, is applicable to several of the pulsed power MIF concepts. Several energy approaches are being studied. Stabilized, pulsed compression using a circulating liquid metal similar to the early Linus concept is one approach [25]. Low-cost refabrication of electrical leads together with a liquid blanket as proposed in the 1979 LASL Conceptual Fast Liner Reactor Study is another. Stand-off delivery of power by plasma jets, lasers, ion beams, or electron beams is a third. Table 1 (above) summarizes how present concepts and efforts fall with respect to different reactor issues and characteristics.

- Science-The intermediate density and pressure regime in which MIF resides, which differs by several to as much as 5-6 orders of magnitude from both MCF and ICF, requires a detailed understanding of the behavior of energy, particle and field transport in high beta plasmas. Flux compression enables the generation of extreme magnetic fields in systems with currents presently available. Can we compress fields to $>100$ Megagauss? Ultrahigh magnetic fields change the properties of the matter in surprising and often hard-to-predict ways. The Magneto-Rayleigh-Taylor instability is a key issue which we address in liners. Magnetized high energy density laboratory plasma physics (MHEDLP) is a relatively unexplored and intellectually rich plasma regime, which is ripe for near-term discoveries, and has also been identified as one of four "cross cutting areas of HEDP of interest to the missions of Federal agencies" [42]. In addition, significant overlap exists with other areas of inquiry, including materials science at high pressures, and the basic science of astrophysics. MHED plasmas that are large compared to the ion gyroradius, at multi-keV temperatures, are enabled in the laboratory by MIF. Recent experiments on MagLIF at Z/Z beamlet have seen large DT/DD fusion yield ratios that are 
Table 1 Functionalities and features of conceptual MIF fusion power cores

\begin{tabular}{ll}
\hline $\begin{array}{l}\text { Target plasma formation } \\
\text { External }\end{array}$ & GF, NRL Linus [25], AFRL/LANL, MSNW/Helion [39] \\
In situ & LANL FLR [26], SNL Z-IFE [40, 41], SNL MagLIF, PJMIF \\
Target plasma type & \\
FRC & NRL Linus, AFRL/LANL, MSNW/Helion \\
Spheromak & GF, PJMIF \\
Z pinch & Flow-stabilized or staged Z Pinch \\
Other & SNL MagLIF, standoff high- $\beta$ [43] \\
Heating & \\
Solid liner compression & LANL FLR, Helion \\
Liquid liner compression & NRL Linus, GF \\
Stand-off & LANL/Hyper-V PJMIF \\
Fusion yield (GJ): rep rate (Hz) & SNL, GF, PJMF \\
Chamber wall protection & \\
Dry wall (none) & LANL/Hyper-V PJMIF (TBD) \\
Thin liquid wall (film) & SNL MagLIF (TBD) \\
Thick liquid wall & NRL Linus, LANL FLR, GF, PJMIF, SNL Z-IFE \\
Sacrificial components/removal of debris & \\
None & GF, NRL Linus, PJMIF \\
Cartridge (leads, coils, etc.) & LANL FLR, SNL Z-IFE, SNL MagLIF, AFRL/LANL \\
\hline
\end{tabular}

strongly suggestive of magnetized ions in the compressed Deuterium plasma.

\section{Near Term ( $\leq 5$ years)}

Near-term research should focus on continuing to explore the science of MIF and to demonstrate quantitative understanding of plasma lifetime, heat and field loss, mix, and implosion physics. Research is also needed on efficient drivers capable of both peak and average power such as linear transformer drivers (LTDs). Magnetized targets need continued improvements in pre-compression lifetime and density for virtually all MIF concepts with microsecondscale or slower implosions. For robust performance, the energy confinement time of the pre-compression target should be an order of magnitude longer than the implosion time. While dedicated and focused efforts are needed for improving target parameters, any effort must also consider compatibility of the target formation and delivery with the specific driver, at all steps of the $R \& D$ effort. There is renewed interest in magnetized ICF by both LLE and LLNL, and finally a standoff plasma liner driver concept has received much theoretical/modeling attention in recent years and is ready for experimental investigations.

For the more mature integrated concepts such as the LANL/AFRL solid liner/FRC or Sandia's MagLIF, the highest priority near-term scientific issues are well defined. The highest priority for the LANL/AFRL effort is to improve the target lifetime and density by factors of 2-3 for better mating with the $\sim 10-\mu$ s implosion time of the solid liner on the Shiva Star capacitor bank. A proposal to do this via merging of twin high performance FRC's has been developed. For MagLIF, integrated implosions with meaningful neutron yield have already been carried out, and a more quantitative understanding of the physics, especially target pre-heat and early mix, B field and thermal energy loss during implosion and acceleration/deceleration-phase interfacial instabilities/mix, is needed. It will also be important to see how target performance behaves with increased preheat laser energy, gas density, axial B field and $\mathrm{Z}$ current for continued performance improvement. Experiments addressing the laser preheat stage are now carried out at multiple laser facilities, including Omega-EP with plans underway at NIF. Sandia is also collaborating with Rochester LLE on the ARPA-E ALPHA program that will include integrated MagLIF experiments on the Omega laser facility.

Although no experiments have been performed to date, simulations indicate that if NIF implosions are near to achieving ignition, magnetizing the fuel may be beneficial. At the high-density regimes of ICF, the main benefits differ somewhat from those of lower-density MIF concepts. For magnetized ICF, a magnetic field provides modest benefits simultaneously in several respects, such as electron thermal insulation, alpha particle trapping, and reduction of instability driven mix. Dedicated efforts to explore a much larger target design space and focused experiments to validate the beneficial physics are needed to fully exploit these physics benefits in integrated shots. Magnetic field coils already exist at LLE/ OMEGA and a prototype is under design/construction at 
LLNL/NIF, thus there are good prospects for near-term advances in magnetized ICF. Limited experiments on Omega where hohlraums have been "magnetized" have also shown improved laser coupling and a reduction in laser-plasma instabilities (LPI) such as Stimulated Raman Scattering. These improvements are likely due to modifications in the electron density and temperature of the under-dense plasma within the hohlraums.

MIF would also benefit significantly from a standoff, high-repetition-rate driver, which would improve the chances for an economic MIF-based fusion reactor. The use of a dynamically formed imploding spherical plasma liner has received attention recently [43]. The science and technology are ready for initial experiments to demonstrate the feasibility of forming imploding plasma liners via merging supersonic plasma jets, and to explore the ram pressure scaling and uniformity of these liners in order to assess their potential as a standoff MIF driver. The PLX facility at LANL has the needed infrastructure, including a $9^{\prime}$ diameter spherical vacuum chamber, multiple diagnostics, and a good portion of the needed capacitors, to carry out 36-60 jet experiments. Accompanying studies on standoff-driver compatible, high- $\beta$ targets could also be initiated, e.g., laser beat-wave magnetization [44]. As mentioned above further development and demonstration of LTD's is needed.

Many of the techniques being proposed for MIF are Rayleigh-Taylor unstable in the final compression. These include the spherical compressions of General Fusion and plasma liners, and the inner surface of the MagLIF liner. The growth of perturbations at the interface between a fluid driver and the buffer magnetic field surrounding the plasma target occurs rapidly in the last few diameters of the implosion, and is not overcome by simply imploding faster [45]. Stabilized liquid liner implosions were demonstrated at the Naval Research Laboratory in the seventies [46], including complete stabilization of liquid liners by a combination of free-piston drive, using high pressure gas, and rotational stabilization of the inner liner surface [25]. The latter technique, now referred to as the stabilized liner compressor (SLC) was demonstrated to provide repetitive cycles of stable, reversible exchange of energy between the compressed payload and the driver gas. This offers the opportunity to achieve repetitive megagauss-level operation while avoiding the "kopek" problem of replacing solid-density liners and their associated connections. The thick, rotating liquid liners provide the replenished firstwall and blanket in reactor concepts. Advances in material strength since the time of the NRL experiments now offer the opportunity for much higher drive pressure (25 vs $3 \mathrm{kpsi}$ ) and faster speeds for the liner compression of a target plasma. Recent funding of the SLC by ARPA-E [38] can permit the return of Linus for the development of plasma targets and the desired power reactor [25].

NNSA sponsors the MagLIF efforts at Sandia. Higher performance MagLIF implosion experiments (after present optimization testing) need the Z-Beamlet laser energy upgrades to $6-8 \mathrm{~kJ}$ of $0.532 \mu \mathrm{m}$ light, axial B fields to $30 \mathrm{~T}$ and $\mathrm{Z}$ current increased to $25 \mathrm{MA}$ to be completed. Improved diagnostics are also required. Upon completion of these tests, good understanding and favorable results would motivate a series of near-break-even (DT equivalent fusion energy release equal to thermal energy in the imploded fuel) tests within the next decade with the Sandia Z-machine for MagLIF or with Los Alamos explosively-driven pulsed power generators using solid liners and FRCs or other suitable plasma formation schemes. The Canadian company General Fusion has accelerated spheromak targets that should be suitable for shock-free compression tests, using electromagnetic (rather than explosively) driven liners. An ignition-class laser driven MIF experiment could be fielded on NIF. An interesting aspect to MIF is that university-scale experiments (such as the $\mathrm{U}$ of Rochester LLE OMEGA facility, and the UNR Nevada Terawatt Facility) can test some MIF target physics. Success with MIF physics basis in the laboratory would then give strong incentive for expanded work on the technologies needed for energy production.

\section{Near Term $(\leq 10$ years $)$}

With progress in the near-term, credible scientific breakeven attempts (as described above) could be made with the lowerdensity concepts, and ignition attempts could be fielded for dedicated magnetized ICF target designs on NIF. Favorable scientific and technical results would justify facility upgrades aimed to explore regimes with higher fusion gains.

From a development perspective, MIF can be viewed as a broader class of ICF possibilities that are characterized by reduced demands on drivers and target performance, although with the complication of adding the B-fields. Possible MIF embodiments range from FRC or spheromak target plasmas, to MagLIF, to ICF targets with B-fields, to a class of Z-pinch like wall-confined plasmas represented by the Russian MAGO configuration. Imploding plasma liners offer untested possibilities such as composite jets/liners carrying the DT fuel and eliminating the need to separately form a target, liners with shaped profiles, and delivery of additional cold fuel for amplified burn and gain. Heating is possible with liner driven implosions or standoff laser beam or particle beam drivers with reduced power and intensity requirements compared with conventional ICF. Development can proceed rapidly because the necessary scientific studies (including burning plasma physics) 
require no new billion-dollar-class facilities. Furthermore, successful implementation of liquid-wall based reactor concepts also eliminates multi-B \$ materials research and development requirements.

\section{Proponents' and Critic's Claims}

Proponents are excited because MIF offers a potentially affordable and attractive path to burning plasma experiments, open with significant innovation, and an intriguing and generally unexplored possibility for practical fusion energy. MIF allows the possibility of more compact fusion systems, the use of thick liquid blankets (no neutron damage problem), a fresh plasma/wall interface on each pulse, and a lower cost development pathway. MIF strengthens the ICF fusion portfolio because it represents both an extra "knob" on existing targets, and enables fundamentally different approaches. So far no physical limitation has been identified that precludes developing MIF as a practical fusion energy system, and several promising development pathways have been identified. Critics argue that pulsed systems (like conventional ICF and MIF) are unlikely to meet the practical requirements for pulse repetition rate and cost per target, especially in the case of MIF, if it involves replacement of liner hardware on every pulse. There are also technical concerns that high-Z liner material will mix rapidly with the relatively low-density fusion fuel, leading to unacceptably large radiation losses. MIF, having far less total funding invested, is understandably less scientifically mature than conventional MFE and ICF approaches.

\section{Summary}

Magneto-inertial fusion is an exciting and largely unexplored approach to achieving pulsed fusion in the laboratory, by merging features of both magnetic and inertial fusion confinement systems. It reduces the IFE driver power requirements by slowing the compression timescale, while fusing at much higher densities than conventional MFE. Multiple variations are being explored at this time, and continued scientific progress would motivate relatively near term opportunities using MIF approaches to explore burning plasmas in the laboratory.

Open Access This article is distributed under the terms of the Creative Commons Attribution 4.0 International License (http://crea tivecommons.org/licenses/by/4.0/), which permits unrestricted use, distribution, and reproduction in any medium, provided you give appropriate credit to the original author(s) and the source, provide a link to the Creative Commons license, and indicate if changes were made.

\section{References}

1. I.R. Lindemuth, R.C. Kirkpatrick, Parameter space for magnetized fuel targets in inertial confinement fusion. Nucl. Fusion 23, 263 (1983)

2. R.C. Kirkpatrick, I.R. Lindemuth, M.S. Ward, Magnetized target fusion: an overview. Fusion Technol. 27, 201 (2005)

3. I.R. Lindemuth, R.E. Siemon, The fundamental parameter space of controlled thermonuclear fusion. Am. J. Phys. 77, 407-416 (2009)

4. M.M. Basko, A.J. Kemp, J. Meyer-ter-Vehn, Ignition conditions for magnetized target fusion in cylindrical geometry. Nucl. Fusion 40, 59 (2000)

5. J.M. Tacetti, T.P. Intrator, G.A. Wurden, S.Y. Zhang, R. Aragonez, P.N. Assmus, C.M. Bass, C. Carey, S.A. deVries, W.J. Fienup, I. Furno, S.C. Hsu, M.P. Kozar, M.C. Langner, J. Liang, R.J. Maqueda, R.A. Martinez, P.G. Sanchez, K.F. Schoenberg, K.J. Scott, R.E. Siemon, E.M. Tejero, E.H. Trask, M. Tuszewski, W.J. Waganaar, FRX-L: a field-reversed configuration plasma injector for magnetized target fusion. Rev. Sci. Inst. 74, 4314-4323 (2003)

6. J.H. Degnan, D.J. Amdahl, A. Brown, T. Cavazos, S.K. Coffey, M.T. Domonkos, M.H. Frese, S.D. Frese, D.G. Gale, T.C. Grabowski, T.P. Intrator, R.C. Kirkpatrick, G.F. Kiuttu, F.M. Lehr, J.D. Letterio, J.V. Parker, R.E. Peterkin Jr, N.F. Roderick, E.L. Ruden, R.E. Siemon, W. Sommars, W. Tucker, P.J. Turchi, G.A. Wurden, Experimental and computational progress on liner implosions for compression of FRCs. IEEE Trans. Plasma Sci. 36, 80-91 (2008)

7. J.H. Degnan, D.J. Amdahl, M. Domonkos, F.M. Lehr, C. Grabowski, P.R. Robinson, E.L. Ruden, W.M. White, G.A. Wurden, T.P. Intrator, J. Sears, T. Weber, W.J. Waganaar, M.H. Frese, S.D. Frese, J.F. Camacho, S.K. Coffey, V. Makhin, N.F. Roderick, D.G. Gale, M. Kostora, A. Lerma, J.L. McCullough, W. Sommars, G.F. Kiuttu, B. Bauer, S.R. Fuelling, R.E. Siemon, A.G. Lynn, P.J. Turchi, Recent magneto inertial fusion experiments on the field reversed configuration heating experiment. Nucl. Fusion 53, 093003 (2013)

8. O.V. Gotchev, N.W. Jang, J.P. Knauer, M.D. Barbero, R. Betti, C.K. Li, R.D. Petrasso, Magneto-inertial approach to direct-drive laser fusion. J. Fusion Energ. 27, 25-31 (2008)

9. O.V. Gotchev, J.P. Knauer, P.Y. Chang, N.W. Jang, M.J. Shoup III, D.D. Meyerhofer, R. Betti, Seeding magnetic fields for laserdriven flux compression in high-energy-density plasmas. Rev. Sci. Instrum. 80, 043504 (2000)

10. J.P. Knauer, O.V. Gotchev, P.Y. Chang, D.D. Meyerhofer, O. Polomarov, R. Betti, J.A. Betti, J.A. Frenje, C.K. Li, M.J.-E. Manuel, R.D. Petrasso, J.R. Rygg, F.H. Seguin, Compressing magnetic fields with high-energy lasers. Phys. Plasmas 17, $056318(2010)$

11. S.A. Slutz, M.C. Herrmann, R.A. Vesey, A.B. Sefkow, D.B. Sinars, D.C. Rovang, K.J. Peterson, M.E. Cuneo, Pulsed-powerdriven cylindrical liner implosions of laser preheated fuel magnetized with an axial field. Phys. Plasmas 17, 056303 (2010)

12. S.A. Slutz, R.A. Vesey, High-gain magnetized inertial fusion. Phys. Rev. Lett. 108, 025003 (2012)

13. R.D. McBride, M.R. Martin, R.W. Lemke, J.B. Greenly, C.A. Jennings, D.C. Rovang, D.B. Sinars, M.E. Cuneo, M.C. Herrmann, S.A. Slutz, C.W. Nakhleh, D.D. Ryutov, J.P. Davis, D.G. Flicker, B.E. Blue, K. Tomlinson, D. Schroen, R.M. Stamm, G.E. Smith, J.K. Moore, T.J. Rogers, G.K. Robertson, R.J. Kamm, I.C. Smith, M. Savage, W.A. Stygar, G.A. Rochau, M. Jones, M.R. Lopez, J.L. Porter, M.K. Matzen, Beryllium liner implosion experiments on the $\mathrm{Z}$ accelerator in preparation for magnetized liner inertial fusion. Phys. Plasmas 20, 056309 (2013) 
14. A.B. Sefkow, S.A. Slutz, J.M. Koning, M.M. Marinak, K.J. Peterson, D.B. Sinars, R.A. Vesey, Design of magnetized liner inertial fusion experiments using the $\mathrm{Z}$ facility. Phys. Plasmas 21, 072711 (2014)

15. M.R. Gomez, S.A. Slutz, A.B. Sefkow, D.B. Sinars et al., Experimental demonstration of fusion-relevant conditions in magnetized liner inertial fusion. Phys. Rev. Lett. 113, 155003 (2014)

16. P.F. Schmit et al., Understanding fuel magnetization and mix using secondary nuclear reactions in magneto-inertial fusion. Phys. Rev. Lett. 113, 155004 (2014)

17. S.C. Hsu et al., Spherically imploding plasma liners as a standoff driver for magneto-inertial fusion. IEEE Trans. Plasma Sci. 40, 1287 (2012)

18. S.C. Hsu et al., Experimental characterization of railgun-driven supersonic plasma jets motivated by high energy density physics applications. Phys. Plasmas 19, 123514 (2012)

19. E.C. Merritt, A.L. Moser, S.C. Hsu, J. Loverich, M. Gilmore, Experimental characterization of the stagnation layer between two obliquely merging supersonic plasma jets. Phys. Rev. Lett. 111, 085003 (2013)

20. E.C. Merritt, A.L. Moser, S.C. Hsu, C.S. Adams, J.P. Dunn, A.M. Holgado, M. Gilmore, Experimental evidence for collisional shock formation via two obliquely merging supersonic plasma jets. Phys. Plasmas 21, 055703 (2014)

21. M. Laberge, an acoustically driven magnetized target fusion reactor. J. Fusion Energ. 27, 65-68 (2008)

22. S. Howard, M. Laberge, L. McIlwraith, D. Richardson, J. Gregson, Development of merged compact toroids for use as a magnetized target fusion plasma. J. Fusion Energ. 28, 156-161 (2009)

23. M. Laberge, Experimental results for an acoustic driver for MTF. J. Fusion Energ. 28, 179-182 (2009)

24. A. G. Es'kov, O. A. Zolotovsky, R. Kh. Kurtmullaev, Ya. N. Laubin, A. J. Malyutin, A. J. Markin, V. N. Semenov, in Experiments on producing the plasma of high-Beta by longitudinal shock waves, Proceedings of the 6th European Conference on Controlled Fusion and Plasma Physics, vol. 1 (Moscow, Russia, 1973)

25. P.J. Turchi, et al, in Review of the NRL liner implosion program, ed. by P.J. Turchi. Megagauss Physics and Technology, (Plenum Press, NY, 1980), p. 375

26. R.W. Moses, R.A. Krakowski, R.L. Miller, in A conceptual design of the fast liner reactor (FLR) for fusion power, LASL Report LA-7686-MS, Feb 1979

27. V.N. Mokhov, V.K. Chernyshev, V.B. Yakubov, et al., in 1979 DAN SSSR vol. 247(1), p. 83 (1979)

28. Oleg M. Burenkov, Yuriy N. Dolin, Pavel V. Duday, Vladimir I. Dudin, Vitaly A. Ivanov, Andrey V. Ivanovsky, Gennady V. Karpov, Andrey I. Kraev,Valery B. Kudelkin, Valentin I. Mamyshev, Ivan V. Morozov, Semen V. Pak, Sergey M. Polyushko, Alexander N. Skobelev, Vladimir A. Tokarev, in New configuration of experiments for MAGO program, Magnetic Field Generation and Related Topics (MEGAGUSS), 2012 14th International Conference on Megagauss, Oct. 14-19, 2012. ISBN 978-4673-5719-7

29. P.J. Turchi, Imploding liner compression of plasma: concepts and issues. IEEE Trans. Plasma Sci. 36(1), 52 (2008)
30. I.R. Lindemuth, R.E. Reinovsky, R.E. Chrien, J.M. Christian, et al., Target plasma formation for magnetic compression/magnetized target fusion, Phys. Rev. Lett., 75(10), 1953-1956 (1995)

31. John Perkins, LLNL, 2015 private communication

32. W. T. Armstrong, J. A. Morgan, in Liner Compression of Magnetically-Confined, FRC Plasma, eds. by C.M. Fowler, R.S. Caird, D.J. Erickson, Megagauss Technology and Pulsed Power Applications, (Plenum Press, NY, 1986). p. 683

33. C. Grabowski, J. Degnan et al., Addressing short trapped-flux lifetime in high-density field-reversed configuration plasmas in FRCHX. IEEE Trans. Plasma Sci. 42(5), 1179 (2014)

34. Y.C.F. Thio, E. Panarella, R.C. Kirkpatrick, C.E.Knapp, F. Wysocki, P. Parks, G. Schmidt, in Magnetized target fusion in a spheroidal geometry with standoff drivers, Current Trends in International Fusion Research, Proceedings $2^{\text {nd }}$ Symposium, Ed. E. Panarella (NRC Canada, Ottawa, Canada, 1999), p. 113

35. Y.C.F. Thio, C.E. Knapp, R.C. Kirkpatrick, R.E. Siemon, P.J. Turchi, J. Fusion Energ. 20, 1 (2001)

36. A.L. Moser, S.C. Hsu, Experimental characterization of a transition from collisionless to collisional interaction between headon-merging supersonic plasma jets. Phys. Plasmas 22, 055707 (2015)

37. S.C. Hsu et al., Laboratory plasma physics experiments using merging supersonic plasma jets. J. Plasma Phys. 81, 345810201 (2015)

38. ARPA-E "ALPHA" program award announcement, 5/14/2015. http://arpa-e.energy.gov/sites/default/files/documents/files/ ALPHA\%20Project\%20Descriptions_FINAL.pdf

39. G. Votroubek, J. Slough, S. Andreason, C. Pihl, Formation of a stable field reversed configuration through merging. J. Fusion Energ. 27(1-2), 123-127 (2008)

40. C. Olson, G. Rochau, S. Slutz, C. Morrow, R. Olson et al., Development path for the Z-Pinch IFE. Fusion Sci. Technol. 47(3), 633 (2005)

41. G.E. Rochau, the Z-Pinch Power Plant Team, Progress toward development of an IFE Power plant using Z-Pinch technology. Fusion Sci. Technol. 47(3), 640 (2005)

42. NNSA FES Interagency Task Force on HEDP, http://www. whitehouse.gov/sites/default/files/microsites/ostp/report_of_the_ interagency_task_force_on_high_energy_density_physics.pdf. Aug. 2007

43. C.E. Knapp, R.C. Kirkpatrick, Possible energy gain for a plasma liner-driven magneto-inertial fusion concept. Phys. Plasmas 21(7), 1 (2014)

44. D. R. Welch et al., in Simulations of magnetic field generation in unmagnetized plasmas via beat-wave current drive, ed. by D.R. Welch et al., PRL 109, 225002; Particle-in-cell simulations of laser beat-wave magnetization of dense plasmas, Physics Plasmas vol. 21, 032704 (2014)

45. P.J. Turchi, Liner stability problems for megagauss fusion. IEEE Trans. Plasma Sci. 43(1(Part II)), 369 (2015)

46. P.J. Turchi et al., Rotational stabilization of an imploding liquid cylinder. Phys. Rev. Lett. 36, 1546 (1976) 